\title{
Optimal Power System Planning with Renewable DGs with Reactive Power Consideration
}

\author{
Hanumesh $^{1}$, Sudarshana Reddy H.R ${ }^{2}$ \\ ${ }^{1}$ Departement of Electrical and Electronics Engineering, Government Polytechnic, Kustagi, Karnataka, India \\ ${ }^{2}$ Departement of Electrical and Electronics Engineering, University B. D .T College of Engineering, Davanagere, India
}

\begin{tabular}{l}
\hline \hline Article Info \\
\hline Article history: \\
Received Aug 9, 2017 \\
Revised Jan 29, 2018 \\
Accepted Feb 14, 2018 \\
\hline
\end{tabular}

\section{Keyword:}

Cuckoo search algorithm Distribution generation Particle swarm optimization Power system planning

\begin{abstract}
This paper analyses the optimal power system planning with DGs used as real and reactive power compensator. Recently planning of DG placement reactive power compensation are the major problems in distribution system. As the requirement in the power is more the DG placement becomes important. When planned to make the DG placement, cost analysis becomes as a major concern. And if the DGs operate as reactive power compensator it is most helpful in power quality maintenance. So, this paper deals with the optimal power system planning with renewable DGs which can be used as a reactive power compensators. The problem is formulated and solved using popular meta-heuristic techniques called cuckoo search algorithm (CSA) and particle swarm optimization (PSO). the comparative results are presented.
\end{abstract}

Copyright (C) 2018 Institute of Advanced Engineering and Science. All rights reserved.

\section{Corresponding Author:}

Hanumesh,

Departement of Electrical and Electronics Engineering,

Government Polytechnic, Kustagi, Karnataka, India

Email: hanumeshsagar138@gmail.com

\section{INTRODUCTION}

Expansion planning of power system with renewable resources for placing DGs in future for satisfying the increase in demand is proposed by Partha kayal in 2014 with multi-objective consideration. Raj Kumar in 2011 discussed about the multi-objective based planning of DG placement. Using impact indices does this. The bus which give more impact for DG placement is identified and placed there. [1,2]. Generator dispatch problems are solved with multi objective in [3]. Placement of wind and solar alone with its mathematical static model is implemented by kayal [4]. This improves the voltage stability and power loss minimization. The Evolutionary algorithms plays an important role in placement problems as it is easy to consider more constraints and fast solution. [5,6]. Previous works deals with only real power [7]. In this paper cuckoo search algorithm [8] is used for the first time in optimal expansion planning of DGs with renewable energy resources with reactive power consideration. And the problem of the minimization is solved by Partha kayal [1] is modified with solving method by using CSA and PSO to get better voltage profile and voltage stability factor and reduced loss. This paper is organised as follows, Section II talks about the the problem formulation.Section III talks about the Pseudo code and Flowchart,Section IV talks about the Results and Discussion, followed by the Conclusion and References. 


\section{PROBLEM FORMULATION}

(i) Total cost of renewable DGs due to installation, operation and maintenance,

$$
\operatorname{Cost}_{D G \text { ren }}=\sum_{i=2}^{N} \sum_{j \in t y p e} I C_{i j} * n_{i} * l_{i}+\left(\sum_{i=2}^{N} \sum_{j \in t y p e} O M C_{i j} * P_{D G \text { ren } i j} * n_{i} * l_{i}\right) * C P V
$$

Where

$I C_{i j}-$ Inverstment cost type 'j'renewable DGat bus ' $i$ '

$O M C_{i j}$ - Operation and maintenance cost of type 'j'renewable DGat bus ' $i$ '

$n_{i}-$ number of $D G$ unit connected at bus ' ${ }^{\prime}$ '

$l_{i}-$ location variable at bus ' $i$ ' $(0$ or 1$)$

$P_{D G \text { ren } i j}$ - Power generated by type ' $j$ ' DG at bus ' $i$ '

$N-$ number of buses in the network

$C P V$ - cumulative present value

$$
C P V=\frac{\left(1-P V^{N}\right)}{(1-P V)}
$$

Here,

Present value of cost

$$
P V=\frac{1+\frac{I F}{100}}{1+\frac{I R}{100}}
$$

Where,

IF - Inflation rate

$I R$ - interest rate

$N_{y}-$ Number of year in planning horizon

(ii) Total benefit that can be achieved from operation of distribution network with renewable DGs is given by,

Benefit $_{D G \text { ren }}=\left\{\left(\sum_{i=2}^{N} \sum_{j \in \text { type }}\left(P_{D G \text { ren } i j}+Q_{D G \text { ren } i j}\right) * n_{i} * l_{i}\right)+\Delta P \operatorname{loss}_{D G \text { ren }}\right\} * C_{h r} * 8760 * C P V$

$\Delta$ Ploss $_{D G \text { ren }}-$ Power loss due to allocation of renewable DGs

$C_{h r}-$ Cost of electricity

Benefit to cost ratio

$$
B C R_{D G \text { ren }}=\frac{\text { Benefit }_{D G \text { ren }}}{\text { Cost }_{D G \text { ren }}}
$$

DISCO benefited more when $B C R_{D G \text { ren }}>1$

(iii) Voltage stability factor

Due to the placement of DGs in power system changes the voltage profile so there is a need for improving the voltage profile, the below equation shows the VSF for any bus i+lin the distribution network,

Here,

$$
V S F_{i+1}=\left(2 V_{i+1}-V_{i}\right)
$$

$V_{i}$ - voltage magnitude at bus $i$

$V_{i+1}-$ voltage magnitude at bus $i+1$

VSF for the entire network is given by

$$
V S F=\frac{\sum_{i=1}^{N-1} V S F_{i+1}}{(N-1)}
$$

(iv) Network security index

Security of the network also should be considered on placement of DG 


$$
L L_{i}=\frac{L_{M V A, i}}{L_{M V A_{\max , i}}}
$$

Network security index can be formulated as below

$$
N S I=\frac{\sum_{i=1}^{N-1} L L_{i}}{(N-1)}
$$

Low value is better.

So,

the objective function is represented as

$$
\text { minimize } f\left(P_{D G \text { ren } i j}, n_{i}, l_{i}\right)=-B C R-V S F+N S I
$$

with respect to equality constraints,

$$
\begin{gathered}
P_{\text {slack }}+\left(\sum_{i=2}^{N} \sum_{j \in \text { type }} P_{D G \text { ren } i j} * n_{i} * l_{i}\right)-\sum_{i=2}^{N} P_{D, i}-P_{L}=0 \\
Q_{\text {slack }}-\sum_{i=2}^{N} Q_{D, i}-P_{L}=0
\end{gathered}
$$

Inequality constraints,

Generation limit at bus i

$$
\begin{aligned}
& P_{D G \text { ren } i j} * n_{i \min } \leq P_{D G \text { ren } i j} * n_{i} \leq P_{D G \text { ren } i j} * n_{i \max } \\
& Q_{D G \text { ren } i j} * n_{i \text { min }} \leq Q_{D G \text { ren } i j} * n_{i} \leq Q_{D G \text { ren } i j} * n_{i \max } \\
& \text { Here } Q_{D G \text { ren } i j}=P_{D G \text { ren } i j} * \tan \left(\cos ^{-1}(P F)\right)
\end{aligned}
$$

Bus voltage tolerance constraint at bus i

$$
V_{i, \min } \leq V_{i} \leq V_{i, \max }
$$

Line capacity constraint of line connecting bus $\mathrm{i}$ and bus $\mathrm{j}$

$$
S_{i j} \leq S_{i j}^{\max }
$$

Where $S_{i j}$ and $S_{i j}{ }^{m a x}$ are actual and maximum line power flow in MVA

Both Particle Swarm Optimization and the Cuckoo Serch Algorithms are used for the solution which is explained in the form of flowchart abd pseudo code in the following section.

\section{PSEUDO CODE AND FLOWCHART}

The PSO technique the position of each particle corresponds to the solution variables which get updated in each particle movement. A comprehensive pseudo code of PSO based OPF is given below, For each particle $\mathrm{i}=1 \ldots . . . \mathrm{S}$

a. do:

b. Initialize the particle's position with a uniformly distributed random vector: $x i \sim$ U(blo,bup), where blo and bup are the lower and upper boundaries of the search-space.

c. Initialize the particle's best known position to its initial position: pi $\leftarrow x i$.

d. If $(\mathrm{f}(\mathrm{pi})<\mathrm{f}(\mathrm{g}))$ update the swarm's best known position:g $\leftarrow$ pi.

e. Initialize the particle's velocity: vi U(-|bup-blo|,|bup-blo|).

f. Until a termination criterion is met (e.g. number of iterations performed, or a solution with adequate objective function value is found), repeat:

g. For each particle $\mathrm{i}=1, \ldots, \mathrm{S}$ do:

h. For each dimension $\mathrm{d}=1, \ldots, \mathrm{n}$ do:

i. Pick random numbers: $\mathrm{rp}, \mathrm{rg} \sim \mathrm{U}(0,1)$

j. $\quad$ Update the particle's velocity: vi,d $\leftarrow \omega v i, d+\varphi p r p(p i, d-x i, d)+\varphi g \quad \operatorname{rg}(g d x i, d)$

k. Update the particle's position: $\mathrm{xi} \leftarrow \mathrm{xi}+\mathrm{vi}$

1. If ( $(\mathrm{f}(\mathrm{xi})<\mathrm{f}(\mathrm{pi})) \mathrm{do}$ :

m. Update the particle's best known position: pi $\leftarrow$ xi.

Int J Pow Elec \& Dri Syst, Vol. 9, No. 2, June 2018 : 750 - 756 
n. If $(\mathrm{f}(\mathrm{pi})<\mathrm{f}(\mathrm{g}))$ update the swarm's best known position: $\mathrm{g} \leftarrow$ pi.

o. Now $\mathrm{g}$ holds the best found solution.

p. The parameters $\omega, \varphi \mathrm{p}$, and $\varphi \mathrm{g}$ are selected by the practitioner and control the behaviour and efficacy of the PSO method,

For simplicity, flow chart of PSO algorithm is given in Figure 1

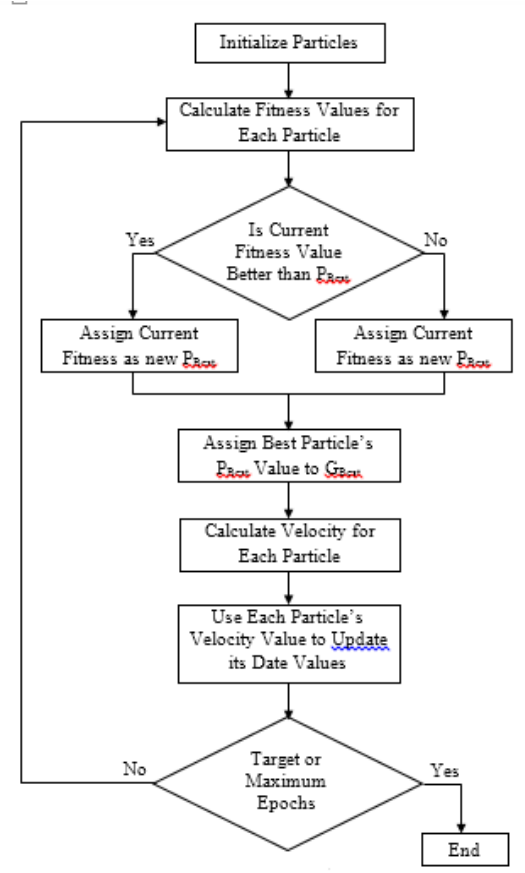

Figure 1. Flowchart of Particle Swarm Optimization

Cuckoo bird lays eggs in communal nests. If the host bird finds the different eggs in its nest it may push down the eggs or it may abandon the nest and build new nest. The cuckoo bird selects the nest of host to lay eggs with similarity in shape and color of eggs to that of host eggs. This increases the probability of hatching cuckoo eggs. And also, cuckoo bird's eggs hatches faster compared to host bird eggs. And also, chicks can mimic the host bird sound for getting food from it. This concept is made as mathematical equation and the concept of search of levy flights which make 90 degrees turn leading to scale free search makes this algorithm more reliable. The procedure of Cuckoo search algorithm is shown as below.

For simplicity in describing new Cuckoo Search, we now use the following three idealized rules:

1) Each cuckoo lays one egg at a time, and dump its egg in randomly chosen nest;

2) The best nests with high quality of eggs will carry over to the next generations;

3) The number of available host nests is fixed, and the egg laid by a cuckoo is discovered by the host bird with a probability pa $\in[0,1]$.

In this case, the host bird can either throw the egg away or abandon the nest, and build a completely new nest. For simplicity, this last assumption can be approximated by the fraction pa of the $\mathrm{n}$ nests are replaced by new nests (with new random solutions

The pseudo code for cuckoo algorithm is briefly given below:

begin

Objective function $\mathrm{f}(\mathrm{x}), \mathrm{x}=(\mathrm{x} 1, \ldots, \mathrm{xd}) \mathrm{T}$

Generate initial population of

$\mathrm{n}$ host nests xi $(\mathrm{i}=1,2, \ldots, \mathrm{n})$

while ( $\mathrm{t}<$ MaxGeneration) or (stop criterion)

Get a cuckoo randomly by L'evy flights

evaluate its quality/fitness $\mathrm{Fi}$

if $(\mathrm{Fi}>\mathrm{Fj})$,

Choose a nest among $n$ (say, j) randomly

replace $\mathrm{j}$ by the new solution;

Optimal Power System Planning with Renewable DGs with Reactive Power Consideration (Hanumesh) 
end

A fraction (pa) of worse nests are abandoned and new ones are built;

Keep the best solutions (or nests with quality solutions);

Rank the solutions and find the current best

end while

Post process results and visualization

end

The flow chart cuckoo algorithm is given below:

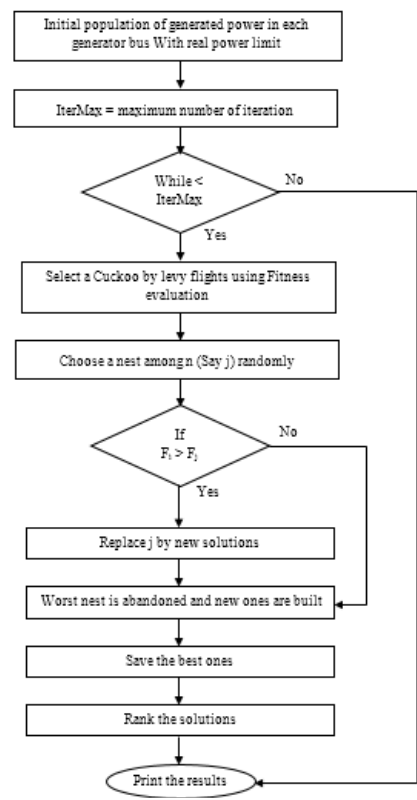

Figure 2. Flowchart of Cuckoo Search Algorithm

\section{RESULTS AND ANALYSIS}

The test system used here is Indian 28 distributed bus system [2] (appendix II). The minimization of Equation (10) is implemented with PSO algorithm and CSA algorithm. The number of DG installed is taken constant $(\mathrm{N}=6)$ as 6 . And the problem is solved for 10 years $(\mathrm{yr})$ duration $\left(\mathrm{N}_{\mathrm{yr}}=10\right)$. The types of DGs are chosen as Solar, Wind and biomass. Here type ' 1 ' is solar, type ' 2 ' is wind and type ' 3 ' is biomass. And it works for reactive power support also. The power factor considered here is 0.9. Appendix 1 shows the parameters used for it [1].

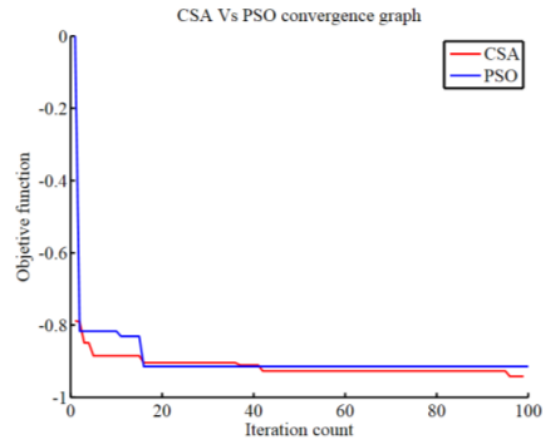

Figure 3. Convergence Graph of CSA and PSO

Int J Pow Elec \& Dri Syst, Vol. 9, No. 2, June 2018 : 750 - 756 
Figure 4 shows the convergence graph of CSA and PSO algorithm with same objective function. Here total number of population is taken as 60 and iteration count as 100 for both the algorithm. The above graph shows the performance of CSA in red line and PSO in blue line. For 100 iteration CSA gives better minimum objective function. Both satisfies the constraints.

Table 1. Results of PSO Algorithm

\begin{tabular}{|c|c|c|c|c|c|c|c|}
\hline Bus & nos. & type & Size (MW) & Size (MVar) & IC in Rs & OMC in Rs/yr & TC Rs/yr \\
\hline 26 & 6 & 3 & 1.2 & 0.6 & 789742.3831 & 121067.5073 & 910809.8904 \\
\hline 3 & 4 & 2 & 0.5 & 0.2 & 159081.0395 & 25083.8983 & 184164.9378 \\
\hline 9 & 3 & 3 & 0.6 & 0.3 & 197435.5958 & 30266.87683 & 227702.4726 \\
\hline 15 & 5 & 3 & 1 & 0.5 & 548432.2105 & 84074.65787 & 632506.8684 \\
\hline 24 & 3 & 2 & 0.375 & 0.2 & 89483.0847 & 14109.6928 & 103592.7775 \\
\hline \multirow[t]{2}{*}{25} & 4 & 3 & 0.8 & 0.4 & 350996.6147 & 53807.78103 & 404804.3957 \\
\hline & & Total & 4.475 & 2.2 & 2135170.928 & 328410.4142 & 2463581.342 \\
\hline
\end{tabular}

Table 2. Results of CSA Algorithm

\begin{tabular}{|c|c|c|c|c|c|c|c|}
\hline Bus & nos. & type & Size (MW) & Size (MVar) & IC in $R s$ & $\mathrm{OMC}$ in $\mathrm{Rs} / \mathrm{yr}$ & TC Rs/yr \\
\hline 14 & 5 & 3 & 1 & 0.5 & 548432.2105 & 84074.65787 & 632506.8684 \\
\hline 20 & 3 & 2 & 0.375 & 0.2 & 89483.0847 & 14109.6928 & 103592.7775 \\
\hline 19 & 4 & 3 & 0.8 & 0.4 & 350996.6147 & 53807.78103 & 404804.3957 \\
\hline 26 & 6 & 3 & 1.2 & 0.6 & 789742.3831 & 121067.5073 & 910809.8904 \\
\hline 13 & 5 & 3 & 1 & 0.5 & 548432.2105 & 84074.65787 & 632506.8684 \\
\hline \multirow[t]{2}{*}{7} & 6 & 2 & 0.75 & 0.4 & 357932.3388 & 56438.77118 & 414371.11 \\
\hline & & Total & 5.125 & 2.5 & 2685018.842 & 413573.0681 & 3098591.91 \\
\hline
\end{tabular}

Table 3. Comparison with PSO Vs CSA

\begin{tabular}{crrrrrrr}
\hline & loss in MW & BCR & VSF & NSI & Final obj & Time in sec & TC in Rs. \\
\hline CSA & 0.0552 & 0.114 & 0.9996 & 0.1711 & -0.9424 & 721.623107 & 3098591.91 \\
PSO & 0.075 & 0.1206 & 0.9996 & 0.2056 & -0.9146 & 174.759907 & 2463581.342 \\
Conventional & 0.044 & 0.1383 & 0.9589 & 0.4024 & - & - \\
method [1] & & & & & &
\end{tabular}

Table 1 shows the results of PSO algorithm with position, numbers, type, IC, OMC and TC. Table 2 shows the results of CSA algorithm with position, numbers, type, IC, OMC and TC. Table 3 shows the comparative results of CSA Vs PSO with TC (Total Cost), Time taken, final objective value, NSI, VSF, BCF and Loss in MW.

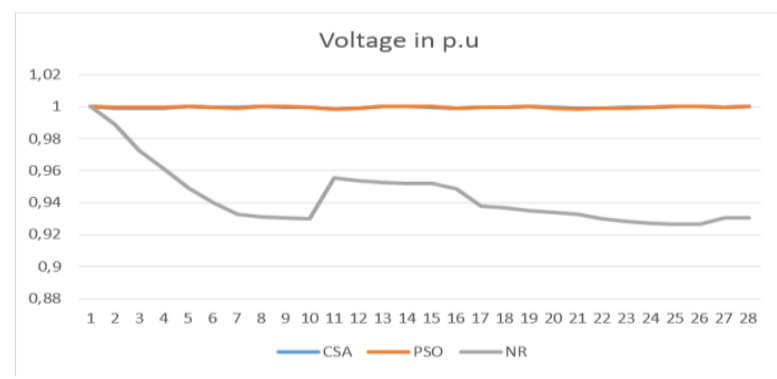

Figure 4. Voltage Profile of Indian 28 Bus System for with Renewable DG Placed Optimally with CSA, PSO and Without Renewable DG with NR (Newton-Raphson) Method

\section{COMPARISON WITH CONVENTIONAL METHOD FROM [1]}

CSA algorithm gives better voltage profile, NSI, VSF and BCR value compared to PSO algorithm and conventional method used in [1]. And vottage profile nears the value of 1 p.u as shown in Figure.4. But 
PSO algorithm gives lesser cost and lesser time taken for solving the algorithm. So, the total cost of the renewable Dgs per year is less in PSO and more in CSA.

\section{CONCLUSION}

The real and reactive power supplied by using DG placement. For Indian 28-bus system expansion planning is conducted for 10-year duration to identify the total cost, Network security index and loss reduction and to improve the voltage stability factor, benefit cost ratio. Traditional PSO algorithm and cuckoo search algorithm is used to solve this problem. And comparatively CSA gives better VSF, NSI and voltage profile compared to PSO. And PSO gives lesser cost and lesser time to solve the problem. So, for implementation choice of algorithm can be chosen based on the real-time requirement.

\section{REFERENCES}

[1] Partha Kayal, Tanushree Bhattacharjee \& Chandan Kumar Chanda, "Planning of renewable DGs for distribution network considering load model: a multi-objective approach", Energy Procedia 54 (2014) 85 - 96

[2] RAJ KUMAR SINGH and S. K. GOSWAMI, "Multi-objective Optimization of Distributed Generation Planning Using Impact Indices and Trade-off Technique" Electric Power Components and Systems, 39:1175-1190, 2011

[3] Guo CX, Bai YH, Zheng X, Zhan JP, Wu QH. "Optimal generation dispatch with renewable energy embedded using multiple objectives". Int. J. of Electr. Power Energy Syst 2012; 42:440-447.

[4] Kayal P, Chanda CK. "Placement of wind and solar based DGs in distribution system for power loss minimization and voltage stability improvement". Int. J. of Electr. Power Energy Syst 2013; 53:795-809.

[5] Kathod DK, Pant V, Sharma J. Evolutionary programming based optimal placement of renewable distributed generators. IEEE Trans. on Power Syst 2013; 28:683-695.

[6] Arya LD, Koshti A, Choube SC. "Distributed generation planning using differential evolution accounting voltage stability consideration". Int. J. of Electr. Power Energy Syst 2012; 42:196-207.

[7] Singaresu S. Rao, "Engineering Optimization: Theory and practice”, Fouth edition, John Wiley \& Sons, Inc. 2009

[8] Xin-She Yang, "Cuckoo Search via Levy Flights", 2009, World Congress on Nature \& Boilogically Inspired Computing (NaBIC).

\section{APPENDIX I}

\begin{tabular}{ccccc}
\multicolumn{4}{c}{ Technical and Economic Data of Wind and Solar based Renewable Dgs } \\
\hline DG technology & $\begin{array}{c}\text { Commercial } \\
\text { size (kW) }\end{array}$ & $\begin{array}{c}\text { Plant } \\
\text { factor (\%) }\end{array}$ & $\begin{array}{c}\text { Investment } \\
\text { cost (Rs/kW) }\end{array}$ & $\begin{array}{c}\text { Operating } \\
\text { and } \\
\text { maintenance } \\
\text { cost (Rs/kW-year) }\end{array}$ \\
\hline $\begin{array}{c}\text { Solar photovoltaic (PV) } \\
\text { Wind turbine } \\
\text { Biomass }\end{array}$ & 20 & 25 & 350000 & 418 \\
\hline & 200 & 20 & 75000 & 11826 \\
\hline
\end{tabular}

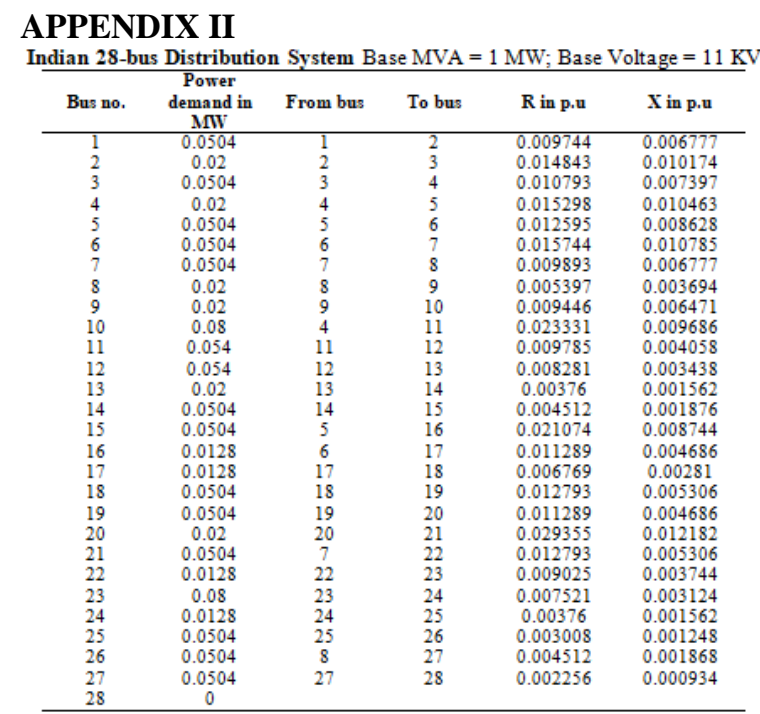

